\title{
Influência do consumo alimentar e da prática de atividade física na prevalência do sobrepeso/obesidade em adolescentes escolares
}

\section{Influence of food consumption and physical activity practice in the prevalence of overweight/obesity in school adolescents}

Erika de Vasconcelos Barbalho ${ }^{*}$ (D), Francisco José Maia Pinto ${ }^{2}$ (D), Francisco Regis da Silva ${ }^{2}$ (D), Rafaella Maria Monteiro Sampaio³ (D), Débora Sâmara Guimarães Dantas² (i)

\author{
'Instituto Superior de Teologia Aplicada (INTA) - Sobral (CE), Brasil. \\ 2Universidade Estadual do Ceará (UECE) - Fortaleza (CE), Brasil. \\ ${ }^{3}$ Universidade de Fortaleza (UNIFOR) - Fortaleza (CE), Brasil.
}

Como citar: Barbalho EV, Pinto FJM, Silva FR, Sampaio RMM, Dantas DSG. Influência do consumo alimentar e da prática de atividade física na prevalência do sobrepeso/obesidade em adolescentes escolares. Cad Saúde Colet, 2020;28(1):12-23. https://doi.org/10.1590/1414-462X202028010181v

\section{Resumo}

Introdução: A adolescência é definida pela Organização Mundial da Saúde como a faixa etária de 10 a 19 anos, pois é nessa fase em que são observadas diferentes mudanças. Objetivo: Analisar a existência de associação entre sobrepeso/obesidade e fatores de risco em adolescentes escolares. Método: Estudo transversal, conduzido nas escolas de nível médio, da rede pública estadual, na cidade de Sobral, no Ceará, com amostra de 572 jovens. Os dados foram coletados utilizando questionário semiestruturado, IPAQ e Marcadores do Consumo Alimentar SISVAN. Na análise não ajustada, utilizou-se da regressão de Poisson, pelo teste do Qui-quadrado, de Wald, com as variáveis que apresentaram nível descritivo $\mathrm{p}<0,20$, como critério de entrada, e, no modelo final, da regressão múltipla de Poisson, apenas com as variáveis significativas $(p<0,05)$. Resultados: No modelo final, por meio da regressão múltipla de Poisson, configuraram-se estatisticamente significativas a idade do adolescente $(p=0,002 ; \mathrm{IC} 95 \%=0,099$; $0,591 ; R P=4,54)$ e a ingestão inadequada de alimentos com alta densidade energética ( $p=0,006$; IC95\% $=0,369 ; 0,848 ; R P=1,79)$ como fatores de risco. Conclusão: Os achados desta pesquisa indicaram que a idade jovem e o consumo inadequado de alimentos são fatores de risco para o desenvolvimento de sobrepeso/obesidade, comprovando sua natureza diversa e complexa.

Palavras-chave: sobrepeso; obesidade; fatores de risco; saúde do adolescente.

\begin{abstract}
Background: adolescence is defined by the World Health Organization, as the age group of 10 to 19 years old, because it is there, when different changes are observed. Objective: to analyze the existence of association between overweight/obesity and risk factors in school adolescents. Method: this is a cross-study, which was performed in high schools belonging to the state public network, in the city of Sobral, Ceará, with a sample of 572 young students. Data were collected using a structured questionnaire, IPAQ, and SISVAN food consumption markers. In the unadjusted analysis, the Poisson regression was used, using the chi-square test of Wald with the variables presenting a descriptive level $p<0.20$ as input criterion, and in the final multiple regression model of Poisson, only the significant variables $(p<0,05)$. Results: in the final model, after performing Poisson multiple regression, adolescent age $(p=0.002 ; \mathrm{Cl}$ $95 \%=0.099 ; 0.591 ; R P=4.54)$ and inappropriate consumption of food with high energy density $(p=0.006$;
\end{abstract}

Trabalho realizado nas escolas de nível médio, da rede pública estadual, na cidade de Sobral (CE), Brasil.

Correspondência: Erika de Vasconcelos Barbalho. E-mail: erikavascb@gmail.com

Fonte de financiamento: nenhuma.

Conflito de interesses: nada a declarar.

Recebido em: Abr. 17, 2018. Aprovado em: Maio 27, 2019

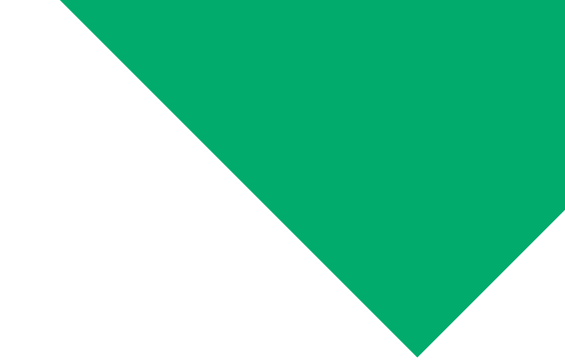


$\mathrm{Cl} 95 \%=0.369 ; 0.848 ; \mathrm{RP}=1.79)$ were configured statistically significant as risk factors. Conclusion: The findings of this research indicated that both young age and inadequate food consumption are risk factors for the development of overweight / obesity. This finding proves that the development of overweight / obesity is diverse and complex.

Keywords: overweight; obesity; risk factors; adolescent health.

\section{INTRODUÇÃO}

A adolescência é definida pela Organização Mundial da Saúde (OMS) como a faixa etária de 10 a 19 anos, pois é nessa fase em que são observadas mudanças fisiológicas, psicológicas e sociais, que podem ocorrer de forma variada entre os indivíduos. Trata-se também de um período de vulnerabilidade nutricional e de consolidação dos hábitos alimentares, que, não sendo adequados, podem resultar no risco de desenvolvimento de diversas doenças.

No mundo, os adolescentes constituem aproximadamente $20 \%$ da população total ${ }^{1,2}$. No Brasil, segundo os dados do Instituto Brasileiro de Geografia e Estatística (IBGE) ${ }^{3}$, no ano de 2010, a população total de adolescentes era pouco mais de 34 milhões de indivíduos, representando cerca de $17 \%$ da população.

Nesse sentido, é ressaltado que, nas últimas décadas, a obesidade tem se apresentado como uma epidemia global na população jovem, sendo considerada uma importante preocupação em saúde pública por causa, principalmente, das comorbidades associadas a essa patologia ${ }^{4}$. Assim, a OMS define sobrepeso e obesidade como o acúmulo anormal de gordura que representa risco à saúde. Dessa forma, sobrepeso e obesidade são classificados em função da razão entre o peso e o quadrado da altura da pessoa (Índice de Massa Corporal - IMC) ${ }^{5}$.

É evidenciado que, com as crescentes inovações tecnológicas, os adolescentes se dispõem a passar grande parte do seu tempo diariamente usando aparelhos eletrônicos, como televisão, computador e celular, tornando-se cada vez mais adeptos a esses modismos e culminando com comportamento sedentário e desenvolvimento do sobrepeso/obesidade ${ }^{6}$.

O comportamento sedentário e a inatividade física, associados a uma alimentação não balanceada, com alto teor de gorduras e pobre em fibras e frutas, geram sobrepeso e obesidade, condições que estão atingindo valores alarmantes em todo o mundo 7 . Como consequência desses hábitos inadequados, no Brasil, aproximadamente $1 / 5$ dos adolescentes está com excesso de peso ${ }^{8}$. Em Porto Alegre, no Rio Grande do Sul, estudo realizado envolvendo 511 adolescentes encontrou prevalência de $27,4 \%$ de sobrepeso/obesidade 9 . Em uma pesquisa que avaliou 383 estudantes em Cascavel, no Paraná, foram observados $23 \%$ de sobrepeso/obesidade ${ }^{10}$.

No adolescente, isso se torna um risco maior por desencadear, precocemente, complicações metabólicas como dislipidemia, hipertensão arterial e outras comorbidades, influenciando de forma negativa a qualidade de vida e a socialização dos jovens ${ }^{11}$.

O estudo do sobrepeso/obesidade associado aos reais fatores na adolescência torna-se relevante, tendo em vista alterações nutricionais, em ascensão em todo mundo. Desse modo, objetivo foi analisar a existência de associação entre sobrepeso/obesidade e fatores associados à prevalência em adolescentes escolares na cidade de Sobral, no Estado do Ceará.

\section{MÉTODO}

Estudo transversal, descritivo e analítico, conduzido nas escolas de nível médio, da rede pública estadual, na cidade de Sobral, no Ceará, no período de abril a junho de 2015.

Para o cálculo amostral, foram utilizados: população de 10.474 escolares, na faixa etária de 10 a 19 anos; prevalência de sobrepeso/obeso de 17,7\%, com erro amostral de 3\%; e intervalo de confiança de $95 \%$. Participaram da amostra 589 escolares. Inicialmente, foram selecionadas as escolas de ensino regular, composta de 9 escolas urbanas e 5 rurais, e, em seguida, as de ensino profissional, constituídas por 3 escolas urbanas. Em cada uma delas foram selecionados aleatoriamente os estudantes, de ambos os sexos, na faixa etária de 10 a 19 anos de idade, regularmente matriculados, que frequentavam a escola, obedecendo à presença na lista de chamada no dia da coleta. Caso não aceitasse participar da pesquisa, o estudante era substituído por outro, da mesma série e tipo de escola. 
Vinte estudantes da amostra foram excluídos por apresentar alguma limitação física que os impediam de responder aos questionários ou de assumir a posição ortostática, impossibilitando-os da aferição de peso e estatura. Assim, a amostra final foi de 572 estudantes. Essa perda não trouxe prejuízos à pesquisa, tendo em vista que ela foi inferior $10 \%$ da amostra inicialmente encontrada ${ }^{12}$. Dessa perda, 13 estudantes eram da área urbana, e 7, da zona rural (Tabela 1). A justificativa de escolha dessa faixa etária está preconizada para o ensino médio, no qual se concentra a maior prevalência de adolescentes matriculados nesse nível educacional, de acordo com o Censo Demográfico de 2010 realizado pelo IBGE 3 .

Inicialmente, foi solicitada a anuência à Coordenadoria Regional de Desenvolvimento da Educação da 6a região (CREDE 6) para realização do estudo nas escolas públicas estaduais do município de Sobral, no Ceará. Em seguida, foi realizada a visita às escolas sorteadas para apresentação do estudo aos diretores, e, após a sua permissão, fez-se a coleta dos dados.

Posteriormente ao consentimento da direção escolar, foi feito o retorno a cada instituição em visitas consecutivas para proceder ao sorteio das turmas e dos alunos, de acordo com o critério de inserção amostral. Em seguida, foi realizada uma explanação sobre os objetivos da pesquisa e a entrega do Termo de Consentimento Livre e Esclarecido (TCLE) e do Termo de Assentimento. A pesquisa somente foi iniciada após ser submetida ao Comitê de Ética em Pesquisa (CEP) da Universidade Estadual do Ceará (UECE) e aprovada por meio do parecer $n^{\circ}$ 805.758. Portanto, o presente estudo obedeceu às determinações institucionais e à Resolução n 466/2012 do Conselho Nacional de Saúde, que trata das diretrizes e normas regulamentadoras de pesquisas envolvendo seres humanos ${ }^{13}$.

Para a coleta de dados, foi utilizada uma entrevista semiestruturada. As variáveis associativas (fatores associados à prevalência) foram distribuídas em: características socioeconômicas e demográficas (idade, sexo, raça, trabalho, trabalho remunerado, zona de residência, escolaridade do responsável, renda familiar, número de pessoas na casa, tipo de moradia e situação conjugal); atividade física e comportamento sedentário (sedentário/irregularmente ativo e ativo/muito ativo fisicamente), hábitos alimentares (alimentos com baixa densidade energética e alimentos com alta densidade energética) e percepção da saúde (ruim, regular, boa, muito boa e excelente) $)^{10,11}$.

Foram aplicados três formulários semiestruturados: o primeiro, ligado às características socioeconômicas e sociodemográficas e respondidas pelos pais; o segundo, referente aos hábitos alimentares obtidos por meio do Formulário de Marcadores do Consumo Alimentar do Ministério da Saúde (SISVAN), para indivíduos maiores de 5 anos de idade; o terceiro, relativo aos níveis de atividade física e respondidos pelos alunos sob a supervisão do pesquisador. Além disso, aferiram-se as medidas de peso e estatura, que foram úteis para o cálculo do IMC e classificação dos escolares, segundo o estado nutricional ${ }^{5}$.

O consumo alimentar foi avaliado por meio da frequência de consumo, nos sete dias que antecederam à pesquisa, de oito alimentos, grupos de alimentos ou preparações: feijão; hortaliças; frutas in natura; leite; refrigerantes; guloseimas; biscoitos doces; e embutidos. Os quatro primeiros foram considerados alimentos marcadores de alimentação satisfatória, e os quatro últimos, marcadores de alimentação insatisfatória.

Essa classificação foi baseada tanto nas recomendações nutricionais para prevenção de doenças crônicas não transmissíveis quanto em evidências que sugerem a associação dessas variáveis com o excesso de peso e outros fatores associados à prevalência, para doenças crônicas não transmissíveis' ${ }^{14}$.

O Formulário de Marcadores do Consumo Alimentar (SISVAN) identificou os hábitos alimentares dos jovens. Para fins de análise, a frequência de consumo de cada alimento foi classificada como ingestão satisfatória ou insatisfatória, de acordo com as recomendações do Ministério da Saúde ${ }^{14}$.

O nível de atividade física foi avaliado pelo Questionário Internacional de Atividade Física $\left({ }^{(P A Q}\right)^{15}$, versão longa, que avalia o grau de atividade física por meio de informações sobre ocupação, deslocamento para o trabalho e escola, além de atividade física de lazer.

Os resultados foram dicotomizados para efeito de análise dos dados: sedentário/irregularmente ativo e ativo/muito ativo fisicamente. Foi considerado sedentário/ 
irregularmente ativo aquele que não realizou nenhuma atividade física, por pelo menos 10 minutos contínuos, durante a semana ou que a praticava de forma irregular. Foi estabelecido como ativo/muito ativo fisicamente aquele que atendia aos seguintes critérios: a) atividade vigorosa em $\geq 5$ dias/semana e $\geq 30$ minutos por sessão; $b$ ) atividade vigorosa em $\geq 3$ dias/semana e $\geq 20$ minutos por sessão + moderada e/ou caminhada em $\geq 5$ dias/semana e $\geq 30$ minutos por sessão; ou c) qualquer atividade somada em $\geq 5$ dias/semana e $\geq 150$ minutos/semana (caminhada + moderada + vigorosa) ${ }^{15}$.

A classificação do estado nutricional pelo IMC foi realizada por meio das curvas de crescimento em percentis (P) para idade de 5 a 19 anos. Nesta pesquisa, foi utilizada a seguinte classificação do desfecho representado por: não sobrepeso/obeso (IMC $\left.\leq \mathrm{P}_{84}\right) \mathrm{e}$ sobrepeso/obeso $\left(\mathrm{IMC} \geq \mathrm{P}_{85}\right)^{5}$.

O peso corporal foi aferido por meio de balanças tipo portátil, marca Sanny, com capacidade máxima de $150 \mathrm{~kg}$, aferidas pelo Instituto Nacional de Metrologia (INMETRO). A estatura foi medida com o estadiomêtro Sanny, com extensão máxima de $210 \mathrm{~cm}$.

Para determinar o peso, os adolescentes ficaram descalços, vestindo roupas leves, posicionados em pé, com o peso igualmente distribuído em ambos os pés. A estatura foi mensurada com o adolescente permanecendo descalço, em posição anatômica, encostando a parte posterior do corpo, e a cabeça posicionada no plano de "Frankfurt", estando em apneia inspiratória no momento da medida ${ }^{16}$. Ambas as medidas foram coletadas pela mesma pesquisadora, sempre com os mesmos equipamentos rotineiramente calibrados.

Foi considerado como desfecho a variável sobrepeso/obeso, indicada para os adolescentes que apresentaram IMC $\geq$ P85, conforme mensuração das condições patológicas utilizadas na classificação do estado nutricional pelo IMC, por meio das curvas em percentis, segundo a OMS5.

Os dados foram processados no programa estatístico Statistical Package for Social Sciences (SPSS), versão 17.0, além do programa Microsoft Excel ${ }^{\circledR}, 2010$, usado no armazenamento e na construção gráfica.

Inicialmente, foi realizada análise inferencial univariada, não ajustada, entre o desfecho e as variáveis associativas, por meio do teste do Qui-quadrado, de Wald, considerando-se como critério de entrada as variáveis com nível descritível $p<0,20$. No modelo ajustado de regressão múltipla final de Poisson, foram consideradas apenas as variáveis significativas $(p<0,05)$. O bom ajuste do modelo foi verificado pelo teste de Hosmer-Lemeshow ${ }^{17}$ como indicativo da diferença não significativa entre as frequências observadas e esperadas.

\section{RESULTADOS}

A amostra final resultou em 572 estudantes, estratificados por zona de residência e tipo de ensino. Embora 454 (79,6\%) adolescentes pesquisados tenham sido classificados pelo IMC como eutróficos, foi observado que $118(20,6 \%)$ apresentaram prevalência de sobrepeso ou obesidade. Entre os pesquisados, $235(41,1 \%)$ consideraram sua saúde como boa e apenas $3(5 \%)$ a indicaram como ruim (Tabela 1$)$.

Acerca dos hábitos alimentares, 515 (90\%) adolescentes tiveram o consumo inadequado de alimentos com baixa densidade energética, ao passo que $364(63,6 \%)$ possuíram consumo inadequado com alta densidade energética. Em relação à classificação da atividade física, predominou o grupo de estudantes com comportamento sedentário/irregularmente ativo para todos os domínios avaliados. Dessa forma, analisados separadamente, foi verificado que $513(89,7 \%)$ adolescentes eram inativos fisicamente no trabalho, na faixa etária de 15 a 19 anos, com $305(53,3 \%)$ na atividade física doméstica, 331 (57,9\%) na atividade relativa ao lazer e 292 (51\%) utilizando a caminhada até o trabalho como meio de transporte e exercício físico (Tabela 1).

Foi encontrada uma renda média em salários mínimos de 2,05, com desvio-padrão de 0,68, sendo o mínimo de 1 salário e máximo de 5,6 salários mínimos (Tabela 2).

Ao realizar a análise não ajustada, foi observado que apenas as variáveis idade do entrevistado $(p<0,001)$ e consumo de alimentos, com alta densidade energética $(p=0,020)$, foram significativas, com significância de $5 \%(R P=4,56$; IC $=1,88 ; 11,02)$. Em relação ao 
Tabela 1. Variáveis dos hábitos alimentares, nível de atividade física, estado nutricional (IMC) e percepção da saúde dos adolescentes. Sobral-CE, 2015.

\begin{tabular}{|c|c|c|}
\hline Variáveis & $\mathbf{N}=572(\%)$ & $\%$ \\
\hline \multicolumn{3}{|l|}{ Hábitos alimentares } \\
\hline \multicolumn{3}{|c|}{ G1 alimentos com baixa densidade energética } \\
\hline Consumo inadequado & 515 & 90,0 \\
\hline Consumo adequado & 57 & 10,0 \\
\hline \multicolumn{3}{|c|}{ G2 alimentos com alta densidade energética } \\
\hline Consumo inadequado & 364 & 63,6 \\
\hline Consumo adequado & 208 & 36,4 \\
\hline \multicolumn{3}{|l|}{ Atividade física } \\
\hline Sedentário/irregularmente ativo & 513 & 89,7 \\
\hline Ativo/muito ativo & 59 & 10,3 \\
\hline \multicolumn{3}{|l|}{ Doméstico } \\
\hline Sedentário/irregularmente ativo & 305 & 53,3 \\
\hline Ativo/muito ativo & 266 & 46,5 \\
\hline \multicolumn{3}{|l|}{ Lazer } \\
\hline Sedentário/irregularmente ativo & 331 & 57,9 \\
\hline Ativo/muito ativo & 241 & 42,1 \\
\hline \multicolumn{3}{|l|}{ Transporte } \\
\hline Sedentário/irregularmente ativo & 292 & 51,0 \\
\hline Ativo/muito ativo & 280 & 49,0 \\
\hline \multicolumn{3}{|l|}{ IMC } \\
\hline Sobrepeso/obeso & 118 & 20,6 \\
\hline Não sobrepeso/obeso & 454 & 79,6 \\
\hline \multicolumn{3}{|l|}{ Percepção da saúde } \\
\hline Ruim & 3 & 5,0 \\
\hline Regular & 112 & 19,6 \\
\hline Boa & 235 & 41,1 \\
\hline Muito boa & 140 & 24,5 \\
\hline Excelente & 82 & 14,3 \\
\hline
\end{tabular}

desenvolvimento do sobrepeso/obesidade e ao consumo de alimentos com alta densidade energética, foi identificada $\mathrm{RP}=1,79$, com IC $=0,369 ; 0,848$ (Tabela 3).

No modelo ajustado, foram significativas com o desfecho as mesmas variáveis da Tabela 3, porém diferentes quanto ao nível de associação $(p=0,002 ; \mathrm{RP}=4,54 ; \mathrm{IC}=0,009 ; 0,591)$. Isso indica que a fase da pré-adolescência ( 10 a 14 anos de idade) tem aproximadamente cinco vezes mais chances de apresentar sobrepeso/obesidade em relação ao jovem na fase da adolescência (15 a 19 anos). Quanto ao desenvolvimento do sobrepeso/obesidade e o consumo de alimentos 
Tabela 2. Estatísticas descritivas das variáveis relacionadas aos adolescentes e aos responsáveis. Sobral-CE, 2015.

\begin{tabular}{lcccccc} 
Variáveis adolescentes & Mínimo & Máximo & Média $(\mathbf{x})$ & $\mathbf{D P}(\mathbf{S})$ & $\mathbf{C V} \%$ & $\mathbf{X} \pm \mathbf{S}$ \\
\hline Idade (anos) & 10 & 19 & 16,3 & 1,1 & 6,2 & $16,3 \pm 1,1$ \\
\hline Peso (kg) & 36,5 & 112,5 & 59,5 & 11,4 & 19,2 & $59,5 \pm 11,4$ \\
\hline Altura (m) & 1,40 & 1,86 & 1,63 & 0,89 & 54,6 & $1,63 \pm 0,89$ \\
\hline IMC & 13,5 & 38,0 & 22,1 & 3,77 & 17,1 & $22,1 \pm 3,77$ \\
\hline Variáveis responsáveis & & & & & & \\
\hline Renda mensal (SM) & 1 & 5,6 & 2,05 & 0,68 & 33,2 & $2,05 \pm 0,68$ \\
\hline Peso (kg) & 45 & 101,2 & 68,5 & 9,25 & 67,6 & $68,5 \pm 9,25$ \\
\hline Altura (m) & 1,42 & 1,89 & 1,60 & 0,07 & 4,4 & $1,60 \pm 0,07$ \\
\hline IMC & 12,6 & 40,2 & 26,7 & 3,7 & 13,9 & $26,7 \pm 3,7$ \\
\hline PPC
\end{tabular}

DP=Desvio-padrão, CV=Coeficiente de variação.

Tabela 3. Quantidade, percentual, razão de prevalência (RP) não ajustada e intervalo de confiança de $95 \%$ para sobrepeso/obeso, segundo variáveis dos adolescentes e responsáveis. Sobral-CE, 2015.

\begin{tabular}{|c|c|c|c|c|c|c|c|c|c|}
\hline \multirow{2}{*}{$\begin{array}{c}\text { Variáveis } \\
\text { Adolescente }\end{array}$} & \multicolumn{2}{|c|}{ Total } & \multicolumn{2}{|c|}{$\begin{array}{c}\text { Sobrepeso/ } \\
\text { obeso }\end{array}$} & \multicolumn{2}{|c|}{$\begin{array}{c}\text { Não } \\
\text { sobrepeso/ } \\
\text { obeso }\end{array}$} & \multirow{2}{*}{$\begin{array}{c}\begin{array}{c}\text { RP não } \\
\text { ajustada }\end{array} \\
-\end{array}$} & \multirow{2}{*}{$\begin{array}{c}\text { IC95\% } \\
-\end{array}$} & \multirow{2}{*}{$\begin{array}{l}\mathbf{P} \\
- \\
-\end{array}$} \\
\hline & $\mathrm{N}=572$ & $\%$ & $\mathbf{N}$ & $\%$ & $\mathbf{N}$ & $\%$ & & & \\
\hline \multicolumn{10}{|l|}{ Idade } \\
\hline 10 a 14 anos & 21 & 3,67 & 11 & 52,4 & 10 & 47,6 & 4,56 & 1,$88 ; 11,02$ & $<0,001$ \\
\hline 15 a 19 anos & 551 & 96,3 & 107 & 19,4 & 444 & 80,6 & 1 & - & - \\
\hline \multicolumn{10}{|l|}{ Sexo } \\
\hline Feminino & 334 & 58,3 & 66 & 19,8 & 268 & 80,2 & 0,09 & 0,$58 ; 1,32$ & 0,540 \\
\hline Masculino & 238 & 41,6 & 52 & 21,8 & 186 & 78,2 & 1 & - & - \\
\hline \multicolumn{10}{|l|}{ Raça } \\
\hline Branca & 244 & 42,6 & 48 & 19,7 & 196 & 80,3 & - & - & 0,410 \\
\hline Negra & 63 & 11,5 & 17 & 27 & 46 & 73 & - & - & - \\
\hline Parda & 265 & 46,3 & 53 & 20 & 212 & 80 & - & - & - \\
\hline \multicolumn{10}{|l|}{ Trabalha } \\
\hline Sim & 75 & 13,1 & 13 & 17,3 & 62 & 82,7 & 0,78 & 0,$67 ; 2,41$ & 0,440 \\
\hline Não & 497 & 86,8 & 105 & 21,1 & 392 & 78,9 & 1 & - & - \\
\hline \multicolumn{10}{|c|}{ Trabalho remunerado } \\
\hline Sim & 71 & 12,41 & 12 & 16,9 & 59 & 83,1 & 0,76 & 0,$68 ; 2,54$ & 0,470 \\
\hline Não & 511 & 89,3 & 106 & 21,2 & 395 & 78,8 & 1 & - & - \\
\hline \multicolumn{10}{|c|}{ Zona de residência } \\
\hline Urbana & 415 & 72,5 & 90 & 21,7 & 325 & 78,8 & 0,13 & 0,$80 ; 2,04$ & 0,310 \\
\hline Rural & 157 & 27,4 & 28 & 17,8 & 129 & 82,2 & 1 & - & - \\
\hline
\end{tabular}


Tabela 3. Continuação...

\begin{tabular}{|c|c|c|c|c|c|c|c|c|c|}
\hline \multirow{2}{*}{$\begin{array}{c}\text { Variáveis } \\
\text { Adolescente }\end{array}$} & \multicolumn{2}{|c|}{ Total } & \multicolumn{2}{|c|}{$\begin{array}{c}\text { Sobrepeso/ } \\
\text { obeso }\end{array}$} & \multicolumn{2}{|c|}{$\begin{array}{c}\text { Não } \\
\text { sobrepeso/ } \\
\text { obeso }\end{array}$} & \multirow{2}{*}{$\begin{array}{c}\text { RP não } \\
\text { ajustada }\end{array}$} & \multirow{2}{*}{$\begin{array}{c}\text { IC95\% } \\
-\end{array}$} & \multirow{2}{*}{$\begin{array}{l}\mathbf{P} \\
-\end{array}$} \\
\hline & $\mathrm{N}=572$ & $\%$ & $\mathbf{N}$ & $\%$ & $\mathbf{N}$ & $\%$ & & & \\
\hline \multicolumn{10}{|c|}{ Escolaridade do responsável } \\
\hline $\begin{array}{l}\text { Sem } \\
\text { instrução }\end{array}$ & 46 & 8,04 & 9 & 19,6 & 37 & 80,4 & - & - & 0,460 \\
\hline Fundamental & 326 & 56,9 & 62 & 19 & 264 & 81 & - & - & - \\
\hline $\begin{array}{l}\text { Médio/ } \\
\text { superior }\end{array}$ & 200 & 34,9 & 47 & 23,5 & 153 & 76,5 & - & - & - \\
\hline \multicolumn{10}{|l|}{ Renda familiar } \\
\hline $1 \mathrm{SM}$ & 122 & 21,3 & 24 & 19,7 & 98 & 80,3 & - & - & 0,930 \\
\hline 1 a 2 SM & 302 & 52,7 & 64 & 21,2 & 238 & 78,8 & - & - & - \\
\hline$>2 \mathrm{SM}$ & 148 & 25,8 & 30 & 20,3 & 118 & 79,7 & - & - & - \\
\hline \multicolumn{10}{|c|}{ Número de pessoas na casa } \\
\hline Até 4 & 321 & 56,1 & 69 & 21,5 & 252 & 78,5 & 1,13 & 0,$74 ; 1,70$ & 0,560 \\
\hline Acima de 4 & 251 & 43,8 & 49 & 19,5 & 202 & 80,5 & 1 & - & - \\
\hline \multicolumn{10}{|c|}{ Tipo de moradia } \\
\hline Própria & 152 & 26,5 & 29 & 19,1 & 123 & 80,9 & 0,88 & 0,$54 ; 1,39$ & 0,580 \\
\hline Não própria & 420 & 73,4 & 89 & 21,1 & 331 & 78,8 & 1 & - & - \\
\hline
\end{tabular}

Situação conjugal

\begin{tabular}{lccccccccc}
\hline Sem cônjuge & 84 & 14,6 & 15 & 17,9 & 69 & 82,1 & 0,81 & 0,$44 ; 1,47$ & 0,480 \\
\hline Com cônjuge & 488 & 85,3 & 103 & 21,1 & 385 & 78,9 & 1 & - & - \\
\hline Atividade física & & & & & & & & & \\
\hline $\begin{array}{l}\text { Sedentário/ } \\
\text { irregular ativo }\end{array}$ & 331 & 57,8 & 66 & 19,9 & 265 & 80,1 & 0,18 & 0,$60 ; 1,36$ & 0,630 \\
\hline $\begin{array}{l}\text { Ativo/muito } \\
\text { ativo }\end{array}$ & 241 & 42,1 & 52 & 21,6 & 189 & 78,4 & 1 & - & - \\
\hline
\end{tabular}

\section{Consumo alimentar}

\begin{tabular}{|c|c|c|c|c|c|c|c|c|c|}
\hline \multicolumn{10}{|c|}{ G1 alimentos com baixa densidade energética } \\
\hline Adequado & 57 & 9,9 & 11 & 19,3 & 46 & 80,7 & 0,91 & 0,$54 ; 2,19$ & 0,700 \\
\hline Inadequado & 515 & 90 & 107 & 20,8 & 408 & 79,2 & 1 & - & - \\
\hline \multicolumn{10}{|c|}{ G2 alimentos com alta densidade energética } \\
\hline Inadequado & 208 & 36,3 & 57 & 27,4 & 151 & 72,6 & 1,87 & 0,$35 ; 0,80$ & 0,020 \\
\hline Adequado & 364 & 63,6 & 61 & 16,8 & 303 & 83,2 & 1 & - & - \\
\hline
\end{tabular}

com alta densidade energética, foram obtidos $p=0,006$ e RP $=1,79$, com IC $=0,369 ; 0,848$. Isso demonstra que os adolescentes que consomem inadequadamente alimentos com alta densidade energética possuem duas vezes mais chances de ter sobrepeso/obesidade em comparação aos que consomem alimentos adequados (Tabela 4). 
Tabela 4. Modelo final com razão de prevalência (RP) ajustada e fatores associados à prevalência do sobrepeso/ obesidade. Sobral-CE, 2015.

\begin{tabular}{lccc}
\multicolumn{1}{c}{ Variáveis } & RP ajustada & IC a 95\% & Valor $\mathbf{~}$ \\
\hline Idade do adolescente & & & \\
\hline 10 a 14 & 4,54 & 0,$099 ; 0,591$ & $\mathbf{0 , 0 0 2}$ \\
\hline 15 a 19 & 1 & - & - \\
\hline G2 alimentos com alta densidade energética & & & \\
\hline Consumo inadequado & 1,79 & 0,$369 ; 0,848$ & $\mathbf{0 , 0 0 6}$ \\
\hline Consumo adequado & 1 & - & - \\
\hline
\end{tabular}

\section{DISCUSSÃO}

Em geral, há um consenso na literatura quanto à complexidade e à multicausalidade do sobrepeso/obesidade na adolescência. Os fatores mais estudados são os de caráter biológico relacionado ao binômio ingestão de alimentos hipercalóricos e comportamento sedentário ${ }^{18}$.

Embora a maioria dos estudantes tenha apresentado estado nutricional eutrófico de acordo com o IMC, foi verificada prevalência crescente relativa ao sobrepeso/obesidade. A pluralidade acentuada de estudantes nesse estado nutricional caracteriza um grave problema de saúde pública nessa população, conforme dados similares aos de outras pesquisas brasileiras, como os encontrados por Schommer et al..$^{9} \mathrm{em}$ seu estudo de base populacional, em Porto Alegre, envolvendo 511 adolescentes, em que 27,4\% apresentaram excesso de peso. De acordo com Carneiro et al. ${ }^{10}$, ao avaliar 1.169 adolescentes de 26 escolas sorteadas de todas as regiões do município de Goiânia, foi observada uma prevalência de excesso de peso de $21,2 \%$, sendo $14,1 \%$ de sobrepeso e $7,1 \%$ de obesidade, com diferença significativa entre os sexos, com $26,3 \%$ no sexo masculino e $16,8 \%$ no sexo feminino $(p<0,01)$.

Nas últimas quatro décadas, os casos novos de sobrepeso/obesidade juvenil vêm crescendo expressivamente em vários países, como nos Estados Unidos, onde a obesidade nessa faixa etária é considerada um importante problema de saúde pública ${ }^{19}$. Em algumas localidades, como Índia, Inglaterra e Canadá, o desenvolvimento dessas enfermidades está associado a um somatório de complicações adversas e a uma complexa rede de variáveis causadoras $^{20}$.

Nesta pesquisa, foi verificado que o excesso de peso não sofreu alteração com o sexo dos entrevistados. Entretanto, há uma pluralidade na prevalência total de casos de sobrepeso/obesidade em relação ao sexo feminino. Além disso, a faixa etária de 10 a 14 anos foi a que apresentou mais chances de desenvolver essa patologia. Embora ${ }^{20}$ não haja um consenso na literatura acerca dessa associação durante a fase pubertária, o sexo feminino foi o que teve maior tendência quanto ao acúmulo de adiposidade em comparação ao sexo masculino.

No que se refere ao nível socioeconômico, não foi evidenciada associação significativa do desfecho com nenhum quesito investigado, fato semelhante à pesquisa de Mendonça e Farias Junior ${ }^{21}$. Apesar disso, os resultados apontaram que a maioria dos responsáveis tinha renda familiar de 1 a 2 salários mínimos.

Watanabe et al..$^{22}$ referem que a relação entre excesso de peso e fatores socioeconômicos tem grande variação, dependendo do país, da cidade e da faixa etária avaliada. Nos países desenvolvidos, é observado que a prevalência de excesso de peso entre adolescentes é superior entre aqueles pertencentes aos estratos socioeconômicos menos privilegiados.

Nos países em desenvolvimento, o excesso de peso nesses indivíduos tende a ser frequente em famílias com melhores condições socioeconômicas ${ }^{23}$. No entanto, é percebido, no Brasil, que esse crescimento se dá em todos os estratos socioeconômicos, inclusive entre as famílias de baixo poder aquisitivo ${ }^{24}$.

Embora, nesta pesquisa, não tenha sido apresentada associação significativa entre o número de pessoas residentes no mesmo domicílio com a ocorrência do excesso de peso, 
Guedes et al. ${ }^{25}$ consideram que a prevalência de sobrepeso/obesidade diminui de acordo com o aumento no número de pessoas residentes na mesma casa. Aqueles que relataram ser filho único apresentaram $81 \%$ mais chances de apresentar sobrepeso quando comparados aos que relataram possuir cinco ou mais pessoas na casa.

Dessa forma, é evidenciada a influência do nível socioeconômico no crescimento da prevalência relativa ao sobrepeso/obesidade. Porém, a não associação entre essa variável com o desfecho, talvez, tenha acontecido pela falta de informação do responsável quanto à renda familiar e ao tipo de moradia, pois muitos tiveram dificuldade de preencher o item por desconhecimento do assunto ou por indisposição para relatar essas informações.

No que se refere à zona de procedência, conforme estudo realizado pelo Instituto Brasileiro de Geografia e Estatística ${ }^{26}$, a prevalência de sobrepeso/obesidade em adolescentes tende a ser mais alta no meio urbano do que no rural. De forma semelhante, os resultados desta pesquisa corroboram os encontrados por Garibaldi et al. ${ }^{27}$, que evidenciaram a maioria oriunda da zona urbana $(69,8 \%)$. Provavelmente isso se deva ao tipo de alimento consumido na zona rural (produtos in natura), o qual se apresenta diferente daquele consumido na zona urbana (alimentos hipercalóricos), em uma relação direta com o poder aquisitivo dos entrevistados.

Ao investigar a associação entre sobrepeso/obesidade com o nível de instrução do responsável pelos adolescentes, foi verificado que mais da metade declarou ter ensino fundamental completo, porém sem associação significativa. Uma hipótese seria a inconsistência nas respostas de muitos alunos e responsáveis por não terem propriedade sobre essa informação ou pelo fato de que parte dos adolescentes residia e havia sido criada por parentes, e não por pais biológicos.

Os achados de Fonseca et al. ${ }^{15}$ mostram que pais com baixa escolaridade apresentam $o$ risco quase duas vezes mais alto de exceder o peso corporal, sendo as maiores prevalências encontradas naqueles que possuem escolaridade referente ao nível fundamental completo. Em contrapartida, Guedes et al. ${ }^{25}$ constataram que os filhos possuem três vezes mais chances de desenvolver sobrepeso/obesidade quando os pais possuem nível superior.

Ao comparar o IMC entre pais e/ou responsáveis e filhos/escolares, foi ressaltado, embora sem alcançar significância estatística, que mais da metade dos pais e/ou responsáveis foi classificada com estado nutricional de sobrepeso/obesidade. Nesse sentido, segundo Camargo et al..$^{28}$, a condição nutricional do adolescente sofre influência também do ambiente em que vive e da rotina alimentar da família. O risco individual de adquirir excesso de peso é de 2,5 a 4 vezes, se apenas um dos pais é obeso; porém, o risco aumenta para 10 vezes, se o pai e a mãe são obesos ${ }^{29}$.

Ao verificar os domínios da atividade física como fator associado ao sobrepeso/obesidade entre adolescentes, foi notado que o tempo gasto desenvolvendo algum tipo de exercício, tanto no trabalho quanto em casa, como lazer ou transporte, não apresentou associação significativa com o desfecho. No entanto, mais da metade dos adolescentes apresentou comportamento sedentário ou irregularmente ativo para todos os domínios avaliados. O mesmo foi observado por Costa et al. ${ }^{30}$, em que os jovens avaliados tiveram comportamento sedentário. Além disso, Wendpap et al. ${ }^{31}$ indicaram uma prevalência de $70 \%$ referente ao comportamento sedentário em adolescentes.

Outra variável importante que não foi investigada na presente pesquisa, mas que a literatura aborda, é o "tempo de tela", ou seja, o tempo despendido para usar televisão e/ou computador. A premissa é que, quanto maior for o tempo de tela, menor será a prática de atividade física (comportamento sedentário); consequentemente, maior será a prevalência no ganho de peso na adolescência ${ }^{32}$. A recomendação da Academia Americana de Pediatria, para crianças e adolescentes, é não exceder o tempo máximo de 2 horas $/ \mathrm{dia}^{33}$.

As principais barreiras relatadas na literatura que impedem o engajamento dos jovens em atividade física possivelmente advêm de fatores ambientais e socioculturais. A quantidade de tempo que o jovem disponibiliza para as mídias estimula ainda o marketing sobre a ingestão de alimentos industrializados e ricos em açúcares ${ }^{34}$.

Neste trabalho, a maioria dos entrevistados consumiu de forma inadequada alimentos com alta densidade energética e apresentou ingestão insuficiente de alimentos com baixa 
densidade energética, como leite e derivados, frutas, verduras e legumes. Isso é preocupante e corrobora a literatura ${ }^{16}$, uma vez que a má alimentação em jovens é um forte fator de risco para o surgimento do sobrepeso/obesidade entre os adolescentes.

Zanini et al..$^{35}$ acompanharam os hábitos alimentares de 600 adolescentes em Caruaru, no Pernambuco, e identificaram que $90,9 \%$ deles consumiam refrigerantes, $95,4 \%$, doces, e $89,6 \%$, alimentos gordurosos. Bezerra et al. ${ }^{6}$ constataram que os grupos de alimentos com maior percentual de consumo em seu estudo também foram salgadinhos fritos e assados, pizza, refrigerantes e sanduíches, o que condiz com os achados desta pesquisa.

Quanto ao consumo de frutas e verduras, há diversos estudos com resultados semelhantes, como em Muniz et al. ${ }^{36}$, que apresentaram apenas $1 / 3$ dos adolescentes consumindo diariamente frutas ou legumes/verduras. Silva e Silva ${ }^{37}$ também revelaram consumos insuficientes de frutas e vegetais entre adolescentes. No estudo de base escolar de Teo et al. ${ }^{38}$, foi verificada também a inadequação no consumo de frutas e hortaliças entre adolescentes.

Acerca da investigação quanto à percepção de saúde, foi observado nesta pesquisa um índice de percepção regular/ruim entre os estudantes, apesar de haver pluralidade naqueles que consideravam sua saúde como boa, o que foi notado também no estudo envolvendo adolescentes de João Pessoa, na Paraíba ${ }^{21}$. Isso ocorre porque, segundo Agathão et al. ${ }^{39}$, na adolescência os indivíduos experimentam as melhores taxas de saúde e vitalidade. Entretanto, nela é apresentado também um período de risco para a saúde, pela maior independência em relação ao consumo de doces e comportamento sedentário, além da percepção do próprio corpo e de outros fatores agregados.

Os achados desta pesquisa indicaram que a idade jovem e o consumo inadequado de alimentos são fatores associados à prevalência para o desenvolvimento de sobrepeso/obesidade, comprovando sua natureza diversa e complexa.

As limitações desta pesquisa referem-se às dificuldades dos estudantes em relação à leitura e resposta dos pontos de cada questionário, além de não recordarem informações passadas.

É sugerida a adoção de políticas públicas de saúde, a fim de incentivar a promoção da saúde dos adolescentes: hábito da prática regular de atividade física, redução do tempo de exposição às atividades sedentárias e adoção de rotina alimentar saudável. Neste caso, a escola, por ser um local de intenso convívio social, propício para atividades educativas e recreativas, destaca-se como um potencial espaço para a promoção de ações de educação em saúde. Ou seja, a ação intersetorial entre serviços de saúde, escola e comunidade é a base para se desenvolverem ações de conscientização dos jovens para que mantenham hábitos saudáveis e tenham rotinas diárias de atividade física.

\section{REFERÊNCIAS}

1. World Health Organization. Child and adolescent health and development: progress report 2009: highlights. Geneva:WHO; 2010.

2. Castilhos CB, Schneider BC, Muniz LC, Assunção MC. Qualidade da dieta de jovens aos 18 anos de idade, pertencentes à coorte de nascimentos de 1993 da cidade de Pelotas (RS), Brasil. Cien Saude Colet. 2015;20(11):3309-18. http://dx.doi.org/10.1590/1413-812320152011.17822014. PMid:26602709.

3. Instituto Brasileiro de Geografia e Estatística. Censo demográfico: resultados gerais da amostra. Rio de Janeiro: IBGE; 2011.

4. Dumith SC, Muraro MFR, Monteiro AR, Machado KP, Dias M, Oliz MM, et al. Diagnostic properties and cutoff points for overweight prediction through anthropometric indicators in adolescents from Caracol, Piauí, Brazil, 2011. Epidemiol Serv Saude. 2018;27(1):e201715013. http://dx.doi.org/10.5123/s167949742018000100013. PMid:29451609.

5. Organização Mundial da Saúde. Obesity: Childhood overweight and obesity [Internet]. 2009 [citado em 2017 june 20]. Disponível: http://www.who.int/topics/obesity/en/

6. Bezerra IN, Souza AM, Pereira RA, Sichieri R. Consumo de alimentos fora do domicílio no Brasil. Rev Saude Publica. 2013;47(1 Suppl 1):200-11. http://dx.doi.org/10.1590/S0034-89102013000700006. PMid:23703264. 
7. Costa IFAF, Medeiros CCM, Costa FDAF, Farias CRL, Souza DR, Adriano WS, et al. Adolescents: behavior and cardiovascular risk. J Vasc Bras. 2017;16(3):205-13. http://dx.doi.org/10.1590/1677-5449.011816. PMid:29930648.

8. Coelho SEAC, Gubert MB. Insegurança alimentar e sua associação com consumo de alimentos regionais brasileiros. Rev Nutr. 2015;28(5):555-67. http://dx.doi.org/10.1590/1415-52732015000500010.

9. Schommer VA, Barbiero SM, Cesa CC, Oliveira R, Silva AD, Pellanda LC. Excesso de peso, variáveis antropométricas e pressão arterial em Escolares de 10 a 18 Anos. Arq Bras Cardiol. 2014;102(4):312-8. http://dx.doi.org/10.5935/abc.20140038. PMid:24676224.

10. Carneiro CS, Peixoto MDRG, Mendonça KL, Póvoa TIR, Nascente FMN, Jardim TSV, et al. Excesso de peso e fatores associados em adolescentes de uma capital brasileira. Rev Bras Epidemiol. 2017;20(2):260-73. http://dx.doi.org/10.1590/1980-5497201700020007. PMid:28832849.

11. Brasil. Ministério da Saúde. Secretaria de Vigilância em Saúde. Secretaria de Gestão Estratégica e Participativa. Vigitel Brasil 2009: vigilância de fatores de risco e proteção para doenças crônicas por inquérito telefônico. Brasília: Ministério da Saúde, 2010.

12. Nascimento RM, Madeiro Leite ÁJ, Almeida NMGS, Almeida PC, Silva CF. Determinantes da mortalidade neonatal: estudo caso-controle em Fortaleza, Ceará, Brasil. Cad Saude Publica. 2012;28(3):559-72. http:// dx.doi.org/10.1590/S0102-311X2012000300016. PMid:22415188.

13. Brasil. Ministério da Saúde. Conselho Nacional de Saúde. Resolução n 466, de 12 de dezembro de 2012 b. Estabelece diretrizes e normas regulamentadoras de pesquisas envolvendo seres humanos. Diário Oficial da União, Brasília, 12 de dezembro de 2012.

14. Francisco PMSB, Azevedo Barros MB, Segri NJ, Alves MCGP, Cesar CLG, Malta DC. Comparação de estimativas para o auto-relato de condições crônicas entre inquérito domiciliar e telefônico - Campinas (SP), Brasil. Rev Bras Epidemiol. 2011;14(1 Suppl 1):5-15. http://dx.doi.org/10.1590/S1415-790X2011000500002. PMid:22002138.

15. Fonseca MJM, França RF, Faerstein E, Werneck GL, Chor D. Escolaridade e padrões de ganho de peso na vida adulta no Brasil: Estudo Pró-Saúde. Rev Panam Salud Publica. 2012;32(5):376-80. http://dx.doi. org/10.1590/S1020-49892012001100008. PMid:23338695.

16. Bertin RL, Malkowski J, Zutter LCl, Ulbrich AZ. Estado nutricional, hábitos alimentares e conhecimentos de nutrição em escolares. Rev Paul Pediatr. 2010;28(3):303-8. http://dx.doi.org/10.1590/S010305822010000300008.

17. Hosmer DW, Lemeshow S. Applied logistic regression. New York: Wiley \& Sons; 2000, 347 p. http://dx.doi. org/10.1002/0471722146.

18. Fortes LS, Amaral AC, Almeida SS, Ferreira ME. Efeitos de diversos fatores sobre o comportamento alimentar de adolescentes. Cien Saude Colet. 2013;18(11):3301-10. http://dx.doi.org/10.1590/S141381232013001100020. PMid:24196895.

19. Pinho L, Flávio EF, Santos SHS, Botelho ACC, Caldeira AP. Excesso de peso e consumo alimentar em adolescentes de escolas públicas no norte de Minas Gerais, Brasil. Cien Saude Colet. 2014;19(1):67-74. http://dx.doi.org/10.1590/1413-81232014191.1968. PMid:24473604.

20. Oliveira PMP, Pagliuca LMMF. Avaliação de tecnologia educativa na modalidade literatura de cordel sobre amamentação. Rev. Esc. Enferm. 2013;47(1):205-12. http://dx.doi.org/10.1590/S0080-62342013000100026. PMid:23515822.

21. Mendonça G, Farias JC Jr. Percepção de saúde e fatores associados em adolescentes. Rev Bras Ciênc Esporte. 2013;17(3):174-80.

22. Watanabe PL, Fontana FE, Silva MP, Mazzardo O, Bacil EDA, Campos W. Associação entre a provocação referente ao peso corporal e a atividade física em adolescentes. Rev Paul Pediatr. 2017;35(3):309-15. http:// dx.doi.org/10.1590/1984-0462/;2017;35;3;00005. PMid:28977290.

23. Pedroni JL, Rech RR, Halpern R, Marin S, Roth LR, Sirtoli M, et al. Prevalência de obesidade abdominal e excesso de gordura em escolares de uma cidade serrana no sul do Brasil. Cien Saude Colet. 2013;18(5):141725. http://dx.doi.org/10.1590/S1413-81232013000500027. PMid:23670470.

24. Leal VS, Lira PI, Oliveira JS, Menezes RC, Sequeira LA, Arruda Neto MA, et al. Excesso de peso em crianças e adolescentes no Estado de Pernambuco, Brasil: prevalência e determinantes. Cad Saude Publica. 2012;28(6):1175-82. http://dx.doi.org/10.1590/S0102-311X2012000600016. PMid:22666821.

25. Guedes DP, Miranda JT No, Almeida MJ, Silva AJRM. Impacto de fatores sociodemográficos e comportamentais na prevalência de sobrepeso e obesidade de escolares. Rev Bras Cineantropom Desempenho Hum. 2010;12(4):221-31. http://dx.doi.org/10.5007/1980-0037.2010V12N4P221. 
26. Instituto Brasileiro de Geografia e Estatística. Ensino: matrículas, docentes e rede escolar. Rio de Janeiro: IBGE; 2013.

27. Garibaldi F, Tornquist D, Tornquist L, Burgos MS. Prevalência de sobrepeso e obesidade em escolares de Santa Cruz do Sul: um comparativo entre zona urbana e rural. Revista Digital. 2014;18(189):1.

28. Camargo APPM, Barros Filho AA, Antonio MÂ, Giglio JS. A não percepção da obesidade pode ser um obstáculo no papel das mães de cuidar de seus filhos. Cien Saude Colet. 2013;18(2):323-33. http://dx.doi. org/10.1590/S1413-81232013000200004. PMid:23358758.

29. Mosca PRF. Obesidade e genética. Revista HCPA. 2012;32(3):318-31.

30. Costa CS, Flores TR, Wendt A, Neves RG, Assunção MCF, Santos IS. Comportamento sedentário e consumo de alimentos ultraprocessados entre adolescentes brasileiros: Pesquisa Nacional de Saúde do Escolar (PeNSE), 2015. Cad Saude Publica. 2018;34(3):1-11. http://dx.doi.org/10.1590/0102-311x00021017. PMid:29538514.

31. Wendpap LL, Ferreira MG, Rodrigues PR, Pereira RA, Loureiro AS, Gonçalves-Silva RM. Qualidade da dieta de adolescentes e fatores associados. Cad Saude Publica. 2014;30(1):97-106. http://dx.doi.org/10.1590/0102311X00082412. PMid:24627017.

32. Prado CV, Rech CR, Hino AAF, Reis RS. Percepção de segurança no bairro e tempo despendido em frente à tela por adolescentes de Curitiba, Brasil. Rev Bras Epidemiol. 2017;20(4):688-701. http://dx.doi. org/10.1590/1980-5497201700040011. PMid:29267753.

33. Victo ER, Ferrari GLM, Silva JP Jr, Araújo TL, Matsudo VKR. Indicadores de estilo de vida e aptidão cardiorrespiratória de adolescentes. Rev Paul Pediatr. 2017;35(1):61-8. http://dx.doi.org/10.1590/19840462/:2017;35;1;00016. PMid:28977318.

34. Polisseni MLC, Ribeiro LC. Exercício físico como fator de proteção para a saúde em servidores públicos. Rev Bras Med Esporte. 2014;20(5):340-4. http://dx.doi.org/10.1590/1517-86922014200502114.

35. Zanini RV, Muniz LC, Schneider BC, Tassitano RM, Feitosa WM, González-Chica DA. Consumo diário de refrigerantes, doces e frituras em adolescentes do Nordeste brasileiro. Cien Saude Colet. 2013;18(12):373950. http://dx.doi.org/10.1590/S1413-81232013001200030. PMid:24263890.

36. Muniz LC, Zanini RV, Schneider BC, Tassitano RM, Feitosa WM, González-Chica DA. Prevalência e fatores associados ao consumo de frutas, legumes e verduras entre adolescentes de escolas públicas de Caruaru, PE. Cien Saude Colet. 2013;18(2):393-404. http://dx.doi.org/10.1590/S1413-81232013000200011. PMid:23358765.

37. Silva DAS, Silva RJS. Associação entre prática de atividade física com consumo de frutas, verduras e legumes em adolescentes do Nordeste do Brasil. Rev Paul Pediatr. 2015;33(2):167-73. http://dx.doi.org/10.1016/j. rpped.2014.09.003. PMid:25887930.

38. Teo CRPA, Taglietti RL, Baptista F, Meneghini VM. Atitude e prática no consumo de frutas e hortaliças entre adolescentes em vulnerabilidade social. Sci Med. 2014;24(3):237-44. http://dx.doi.org/10.15448/19806108.2014.3.17232.

39. Agathão $B T$, Reichenheim ME, Moraes CL. Qualidade de vida relacionada à saúde de adolescentes escolares. Cien Saude Colet. 2018;23(2):659-68. http://dx.doi.org/10.1590/1413-81232018232.27572016. PMid:29412423. 